\title{
Riesz bases of port-Hamiltonian systems ${ }^{*}$
}

\author{
Birgit Jacob ${ }^{1} \quad$ Julia T. Kaiser ${ }^{1} \quad$ Hans Zwart ${ }^{2,3}$
}

\begin{abstract}
The location of the spectrum and the Riesz basis property of wellposed homogeneous infinite-dimensional linear port-Hamiltonian systems on a 1D spatial domain are studied. It is shown that the Riesz basis property is equivalent to the fact that system operator generates a strongly continuous group. Moreover, in this situation the spectrum consists of eigenvalues only, located in a strip parallel to the imaginary axis and they can decomposed into finitely many sets having each a uniform gap.
\end{abstract}

Mathematics Subject Classification: 35P10, 47B06, 47D06, 35L40.

Keywords: Riesz spectral operator, infinite-dimensional linear port-Hamiltonian system, strongly continuous group.

\section{Introduction}

It is well-known that the eigenvectors of a compact self-adjoint operator form an orthonormal basis of the underlying Hilbert space. In the 1960s Dunford and Schwarz [DS71] introduced the more general notion of spectral operators. Further, Curtain [Cur84 analysed discrete spectral operators, i.e., spectral operators with compact resolvent, and the class of Riesz spectral operators was formulated in [CZ95] and extended in GZ01 to characterize also operators with multiple eigenvalues. For Riesz spectral operator its eigenvectors still form a basis, but this basis is assumed to be Riesz basis. Since a Riesz basis is isomorphic to an orthonormal basis, many of the nice properties of compact self-adjoint operators cary over to Riesz spectral operators. For instance, solutions of the abstract differential equation $\dot{x}(t)=A x(t)+B u(t)$, with $A$ a Riesz spectral operator, can still described by an eigenfunction expansion of non-harmonic Fourier series. This enables that many properties of these infinite-dimensional

\footnotetext{
${ }^{*}$ Support by Deutsche Forschungsgemeinschaft (Grant JA 735/13-1) is gratefully acknowledged.

${ }^{1}$ University of Wuppertal, School of Mathematics and Natural Sciences, IMACM Gaußstraße 20, D-42119 Wuppertal, Germany, \{bjacob,julia.kaiser\}@uni-wuppertal.de

${ }^{2}$ University of Twente, Department of Applied Mathematics, 7500 AE Enschede, The Netherlands, h.j.zwart@utwente.nl

${ }^{3}$ Department of Mechanical Engineering, Technische Universiteit Eindhoven, 5600 MB Eindhoven, The Netherlands
} 
systems such as stability, stabilizability and controllability can be characterized in an elegant manner, see e.g. CZ95, CZ20.

In this article, we investigate the Riesz basis property of a special class of infinite-dimensional systems, namely port-Hamiltonian systems on a 1D spatial domain. Here by the Riesz basis property we mean that the associated system operator is a discrete Riesz spectral operator, see Definition 2.2. Port-based network modelling of complex physical systems leads to port-Hamiltonian systems. For finite-dimensional systems there is by now a well-established theory vdS06, EMvdS07, DMSB09. The port-Hamiltonian approach has been extended to the infinite-dimensional situation by a functional analytic approach in Vil07, ZLMV10, JZ12, JMZ15, Aug16, JZ18. This approach has been successfully used to derive simple verifiable conditions for well-posedness LGZM05, Vil07, ZLMV10, JZ12, JMZ15, JK19b, stability [JZ12, AJ14 and stabilization RZLG17, RLGMZ14, AJ14, SZ18, and robust regulation [HP18. The study of the Riesz basis property for infinite-dimensional port-Hamiltonian systems has started with the thesis by Villegas [Vil07, Chapter 4]. Using results on first order eigenvalue problems by Tretter [Tre00a, Tre00b], he obtained a sufficient condition. However, it is not easy to see when this technical sufficient condition is satisfied.

Many systems have a Riesz basis of eigenfunctions, see e.g. GX04, XG03. In the monograph GW19, Section 4.3] B.-Z. Guo and J.-M. Wang study the Riesz basis property for a closely related class of systems, that is, hyperbolic systems of the form $\frac{\partial x}{\partial t}=K(\zeta) \frac{\partial x}{\partial \zeta}+C(\zeta) x$ with $K$ and $C$ diagonal. Note, that (almost) every port-Hamiltonian system on a one dimensional spatial domain can be transformed into a hyperbolic system of this form. However, in general not with a diagonal $C$. Furthermore, the boundary conditions will be more general, see [ZLMV10, JZ12, or the proof of Lemma 5.1. Our main result thus generalizes their theorem GW19, Theorem 4.11]. We remark, that in this situation the notions of Riesz basis of subspaces and Riesz basis with parentheses are equivalent. Moreover, in XW11] the Riesz basis property is investigated for operators perturbed by output feedback.

Our main result shows that a linear infinite-dimensional port-Hamiltonian system on a 1D spatial domain has the Riesz basis property if and only if the system operator generates a strongly continuous group. Important to note here is that we don't need constant coefficients, nor extra assumption. Since the group property is equivalent to a simple matrix condition, our results enable us to very quickly check whether the Riesz basis property holds, see also our example section. Our proof combines methods from complex analysis, differential equations and mathematical systems theory. In particular, we use the fact that every well-posed port-Hamiltonian control system (12) is exactly controllable in finite time. We refer the reader to Section 4 for the definition of exact controllability.

Notation: $X$ and $Y$ are complex and separable Hilbert space. We denote the space of all bounded linear operators from $X$ to $Y$ by $\mathcal{L}(X, Y)$. To shorten notation we write $\mathcal{L}(X):=\mathcal{L}(X, X)$. Throughout this article we assume that 
$A: \mathcal{D}(A) \subset X \rightarrow X$ is a closed, densely defined, linear operator. The set $\sigma(A)$ refers to the spectrum of $A$ and $\sigma_{p}(A)$ to its point spectrum. We denote by $\rho(A):=\mathbb{C} \backslash \sigma(A)$ the resolvent set of $A$, and for each $s \in \rho(A)$ we denote the resolvent of $A$ by $(s-A)^{-1}:=(s I-A)^{-1}$. The growth bound of a $C_{0}$-semigroup $(T(t))_{t \geq 0}$ with generator $A$ is denoted by $\omega_{0}(A)$ and $s(A):=\sup _{s \in \sigma(A)} \operatorname{Re} s$ is the spectral bound of $A . H^{1}\left((0,1) ; \mathbb{C}^{d}\right)$ denotes the first order Sobolev space on the interval $(0,1)$.

\section{Main Result}

In this section we formulate our main result. We start with the definition of discrete Riesz spectral operators.

Definition 2.1. For an operator $A$ on $X$ we call $\gamma \subset \sigma(A)$ a compact spectral set if $\gamma$ is a compact subset of $\mathbb{C}$ which is open and closed in $\sigma(A)$. The spectral projection on the spectral subset $\gamma$ is defined as

$$
E(\gamma)=\frac{1}{2 \pi i} \int_{\Gamma}(s-A)^{-1} d s,
$$

where $\Gamma$ is a closed Jordan curve containing every point of $\gamma$ and no point of $\sigma(A) \backslash \gamma$.

In this article operators with compact resolvent are of particular interest. The spectrum of these operators is a denumerable set of points with no finite accumulation point, c.f. [DS71, Lemma XIX.2]. Furthermore, every point in the spectrum is an eigenvalue which has finite algebraic as well as finite geometric multiplicity, c.f. GGK90, Theorem XV.2.3]. If $\left(s_{n}\right)_{n \in \mathbb{N}}$ is the spectrum of an operator with compact resolvent we write $E_{n}:=E\left(\left(s_{n}\right)\right), n \in \mathbb{N}$.

Definition 2.2. An operator $A$ with compact resolvent and $\sigma(A)=\left(s_{n}\right)_{n \in \mathbb{N}}$ is a discrete Riesz spectral operator, if

1. for every $n \in \mathbb{N}$ there exists $N_{n} \in \mathcal{L}(X)$ such that $A E_{n}=\left(s_{n}+N_{n}\right) E_{n}$,

2. the sequence of closed subspaces $\left(E_{n}(X)\right)_{n \in \mathbb{N}}$ is a Riesz basis of subspaces of $X$, that is, $\operatorname{span}\left(E_{n}(X)\right)_{n \in \mathbb{N}}$ is dense and there exists an isomorphism $T \in \mathcal{L}(X)$, such that $\left(T E_{n}(X)\right)_{n \in \mathbb{N}}$ is system of pairwise orthogonal subspaces of $X$.

3. $N:=\sum_{n \in \mathbb{N}} N_{n}$ is bounded and nilpotent.

Remark 2.3. Discrete Riesz spectral operator are spectral operators in the sense of Dunford and Schwartz DS71. However, we additionally assume that the operator has a compact resolvent, which are discrete operators in the sense of Dunford and Schwartz [DS71, Definition XIX.1]. Every discrete Riesz spectral operator is a Riesz spectral operator in the sense of Guo and Zwart GZ01. and these are again spectral operator in the sense of Dunford and Schwartz. 
In Curtain and Zwart [CZ95] a slightly stronger notion is considered, where all eigenvalues have to be simple. However, they do not require that the operator has a compact resolvent.

Remark 2.4. If a sequence of vectors $\left(x_{n}\right)_{n \in \mathbb{N}}$ in $X$ is a Riesz basis of $X$, that is, there is an isomorphism $T \in \mathcal{L}(X)$, such that $\left(T x_{n}\right)_{n \in N}$ is an orthonormal basis of $X$, then clearly $\left\{\operatorname{span} x_{n}\right\}_{n \in \mathbb{N}}$ is a Riesz basis of subspaces of $X$.

Remark 2.5. If $A$ is a discrete Riesz operator, then clearly $E_{n}$ commute with $A$ and $A$ is equivalent to the infinite matrix

$$
A=\operatorname{diag}\left(A_{1}, A_{2}, \ldots, A_{n}, \ldots\right),
$$

where $A_{n}$ is a square matrix which corresponds to the restriction $\left.A\right|_{E_{n} X}$ of $A$. Then $A=\sum_{n \in \mathbb{N}} i_{n} A_{n} E_{n}$, where $i_{n}$ is the (natural) inclusion operator and $A_{n}$ is identified with $\left.\left(s_{n}+N_{n}\right)\right|_{E_{n}}$.

We consider first order linear port-Hamiltonian systems on a one-dimensional spatial domain of the form

$$
\begin{aligned}
\frac{\partial x}{\partial t}(\zeta, t) & =\left(P_{1} \frac{\partial}{\partial \zeta}+P_{0}\right)(\mathcal{H}(\zeta) x(\zeta, t)), \\
x(\zeta, 0) & =x_{0}(\zeta), \\
0 & =\left[\begin{array}{ll}
W_{1} & W_{0}
\end{array}\right]\left[\begin{array}{l}
(\mathcal{H} x)(1, t) \\
(\mathcal{H} x)(0, t)
\end{array}\right],
\end{aligned}
$$

where $\zeta \in[0,1]$ and $t \geq 0$ and the Assumption 2.6 is fulfilled. Equation (10) describes a special class of port-Hamiltonian systems, which cover in particular the wave equation, the transport equation and the Timoshenko beam as well as coupled systems. For more information we refer to [JZ12, JZ18.

Assumption 2.6. The $d \times d$ Hermitian matrix $P_{1}$ is invertible, $P_{0}$ is a $d \times d$ skew-symmetric matrix, $\left[\begin{array}{ll}W_{1} & W_{0}\end{array}\right]$ is a full row rank $d \times 2 d$-matrix, and $\mathcal{H}(\zeta)$ is a positive $d \times d$ Hermitian matrix for a.e. $\zeta \in(0,1)$ satisfying $\mathcal{H}, \mathcal{H}^{-1} \in$ $L^{\infty}\left(0,1 ; \mathbb{C}^{d \times d}\right)$. Thus, the matrix $P_{1} \mathcal{H}(\zeta)$ can be diagonalized as $P_{1} \mathcal{H}(\zeta)=$ $S^{-1}(\zeta) \Delta(\zeta) S(\zeta)$, where $\Delta(\zeta)$ is a diagonal matrix and $S(\zeta)$ is an invertible matrix for a.e. $\zeta \in(0,1)$. We suppose the technical assumption that $S^{-1}, S$, $\Delta:[0,1] \rightarrow \mathbb{C}^{d \times d}$ are continuously differentiable.

Definition 2.7. Let $P_{0}, P_{1}, \mathcal{H}$ satisfy Assumption 2.6 and $X:=L^{2}\left((0,1) ; \mathbb{C}^{d}\right)$. Then the operator $A: \mathcal{D}(A) \subset X \rightarrow X$ defined by

$$
\begin{gathered}
A x:=\left(P_{1} \frac{d}{d \zeta}+P_{0}\right)(\mathcal{H} x), \quad x \in \mathcal{D}(A), \\
\mathcal{D}(A):=\left\{x \in X \mid \mathcal{H} x \in H^{1}\left((0,1) ; \mathbb{C}^{d}\right) \text { and }\left[\begin{array}{ll}
W_{1} & W_{0}
\end{array}\right]\left[\begin{array}{l}
(\mathcal{H} x)(1) \\
(\mathcal{H} x)(0)
\end{array}\right]=0\right\},
\end{gathered}
$$

is called port-Hamiltonian operator. 
We equip the space $X:=L^{2}\left((0,1) ; \mathbb{C}^{d}\right)$ with the energy norm $\sqrt{\langle\cdot, \mathcal{H} \cdot\rangle}$, where $\langle\cdot, \cdot\rangle$ denotes the standard inner product on $L^{2}\left((0,1) ; \mathbb{C}^{d}\right)$. Due to our Assumption 2.6 the energy norm is equivalent to the standard norm on $L^{2}\left((0,1) ; \mathbb{C}^{d}\right)$.

Furthermore, we emphasize that a port-Hamiltonian operator which generate a $C_{0}$-semigroup is closed and that its resolvent is compact, see Aug16.

Let $A$ generate a $C_{0}$-semigroup $(T(t))_{t \geq 0}$ on $X$. Then a closed subspace $V \subset X$ is called $(T(t))_{t \geq 0}$-invariant, if $T(t) V \subseteq V$ for all $t \geq 0$. In this case the restriction $\left(\left.T(t)\right|_{V}\right)_{t \geq 0}$ is again a $C_{0}$-semigroup with generator $\left.A\right|_{V}$ on $V$, c.f. [CZ95, Lemma 2.5.3]. We say that a set $\left(s_{n}\right)_{n \in \mathbb{N}} \subset \mathbb{C}$ has uniform gap, if $\inf _{n \neq m}\left|s_{n}-s_{m}\right|>0$ for $n, m \in \mathbb{N}$. By $Z^{+}(\zeta)$, we denote the span of the eigenvectors of $P_{1} \mathcal{H}(\zeta)$ corresponding to the positive eigenvalues of $P_{1} \mathcal{H}(\zeta)$ and by $Z^{-}(\zeta)$ the span of the eigenvectors of $P_{1} \mathcal{H}(\zeta)$ corresponding to the negative eigenvalues of $P_{1} \mathcal{H}(\zeta)$.

We are now in the position to formulate our main result.

Theorem 2.8. Let $A$ be a port-Hamiltonian operator and the generator of a $C_{0}$-semigroup. Then the following is equivalent:

1. A is a discrete Riesz spectral operator.

2. $A$ is the generator of a $C_{0}$-group.

3. $W_{1} \mathcal{H}(1) Z^{+}(1) \oplus W_{0} \mathcal{H}(0) Z^{-}(0)=W_{1} \mathcal{H}(1) Z^{-}(1) \oplus W_{0} \mathcal{H}(0) Z^{+}(0)=\mathbb{C}^{d}$.

If one of the equivalent conditions are satisfied, then $\sigma(A)=\sigma_{p}(A)$ lie in a strip parallel to the imaginary axis, the eigenvalues (counted according to the algebraic multiplicity) can be decomposed into finitely many sets each having a uniform gap and $A$ satisfies the spectrum determined growth assumption, that is, $\omega_{0}(A)=s(A)$.

The proof of Theorem 2.8 will be given in Section 5 .

Remark 2.9. It is particularly easy to check if a port-Hamiltonian operator $A$ is the generator of a unitary $C_{0}$-group, c.f. [JMZ15, Theorem 1.1]. This is actually

the case if and only if $\widetilde{W}_{B} \Sigma \widetilde{W}_{B}^{*}=0$, where $\widetilde{W}_{B}:=\left[\begin{array}{ll}W_{1} & W_{0}\end{array}\right]\left[\begin{array}{cc}P_{1} & -P_{1} \\ I & I\end{array}\right]^{-1}$ and $\Sigma:=\left[\begin{array}{ll}0 & I \\ I & 0\end{array}\right]$. In this case $A$ is even a skew-adjoint operator by Stone's Theorem, c.f. [EN00, Theorem VII.3.24], which implies that the normalized eigenvectors form an orthonormal basis of $X$.

\section{Preliminaries on discrete Riesz spectral oper- ators}

Riesz bases of subspace have the following useful characterizations.

Proposition 3.1. [GZ01, Definition 1.4] Let $A$ be an operator with compact resolvent and $\sigma(A)=\left(s_{n}\right)_{n \in \mathbb{N}}$. Then the sequence of subspaces $\left(E_{n}(X)\right)_{n \in \mathbb{N}}$ is 
a Riesz basis of subspaces of $X$ if and only if there exists positive constants $m_{1}$ and $m_{2}$ such that it holds

$$
m_{1}\|x\|^{2} \leq \sum_{n \in \mathbb{N}}\left\|E_{n} x\right\|^{2} \leq m_{2}\|x\|^{2}, \quad x \in X .
$$

Lemma 3.2. Lemma 3.3. Proposition 3.4 and Proposition 3.5 will be useful for the proof of the main result.

Lemma 3.2. Let $A$ be a discrete Riesz spectral operator and $M:=\|N\|$, where $N$ is given by Definition 2.2. Then there exists $C>0$ such that for $s \in \rho(A)$ with $d(s, \sigma(A))>M$ we have

$$
\left\|(s-A)^{-1}\right\| \leq \frac{C}{d(s, \sigma(A))},
$$

where $d(s, \sigma(A))$ denotes the distance from $s$ to the spectrum of $A$.

Proof. Let $\sigma(A)=\left(s_{n}\right)_{n \in \mathbb{N}}, E_{n}, N_{n}, N$ as in Definition 2.2 and $s \in \rho(A)$ with $d(s, \sigma(A))>M$ be arbitrary. Since $E_{n}$ is a spectral projection, it commutes with $A$ and the resolvent of $A$. By the definition of a discrete spectral operator we have

$$
s-A=\sum_{n=1}^{\infty}\left(\left(s-s_{n}\right)-N_{n}\right) E_{n}
$$

and identifying $\left(s-s_{n}\right)-N_{n}$ with the matrix corresponding to $\left.\left(\left(s-s_{n}\right)-N_{n}\right)\right|_{E_{n} X}$ we obtain

$$
(s-A)^{-1}=\sum_{n=1}^{\infty}\left(\left(s-s_{n}\right)-N_{n}\right)^{-1} E_{n} .
$$

Then it holds for $x \in X$

$$
\begin{aligned}
\left\|(s-A)^{-1} x\right\|^{2} & =\left\|\sum_{n \in \mathbb{N}}\left(\left(s-s_{n}\right)-N_{n}\right)^{-1} E_{n} x\right\|^{2} \\
& \leq \sum_{n \in \mathbb{N}}\left\|\left(\left(s-s_{n}\right)-N_{n}\right)^{-1} E_{n} x\right\|^{2} \\
& \leq \sup _{n \in \mathbb{N}}\left\|\left(\left(s-s_{n}\right)-N_{n}\right)^{-1}\right\|^{2} \sum_{n \in \mathbb{N}}\left\|E_{n} x\right\|^{2} \\
& \leq m_{2} \sup _{n \in \mathbb{N}}\left\|\left(\left(s-s_{n}\right)-N_{n}\right)^{-1}\right\|^{2}\|x\|^{2},
\end{aligned}
$$

where $m_{2}$ is the positive constant of the Riesz basis of subspaces $\left(E_{n}\right)_{n \in \mathbb{N}}$, c.f. Lemma 3.1] Using

$$
\left(\left(s-s_{n}\right)-N_{n}\right)=\left(s-s_{n}\right)\left(I-\frac{1}{\left(s-s_{n}\right)} N_{n}\right)
$$


we get

$$
\left(\left(s-s_{n}\right)-N_{n}\right)^{-1}=\frac{1}{\left(s-s_{n}\right)} \sum_{j=0}^{k_{n}} \frac{1}{\left(s-s_{n}\right)^{j}} N_{n}^{j},
$$

where $k_{n}$ denotes the degree of nilpotency of $N_{n}$. Thus, for $s \in \rho(A)$ such that $d(s, \sigma(A))>M$, it holds

$$
\begin{aligned}
\left\|\left(\left(s-s_{n}\right)-N_{n}\right)^{-1}\right\| & \leq \sum_{j=1}^{k_{n}+1} \frac{1}{\left|\left(s-s_{n}\right)\right|^{j}} M^{j-1} \leq \sum_{j=1}^{k_{n}+1} \frac{1}{d(s, \sigma(A))^{j}} M^{j-1} \\
& \leq \frac{1}{d(s, \sigma(A))} \sum_{j=0}^{\infty}\left(\frac{M}{d(s, \sigma(A))}\right)^{j},
\end{aligned}
$$

which concludes the proof.

Lemma 3.3. Let $A$ be a discrete Riesz spectral operator and generator of a $C_{0}$-semigroup, and $P \in \mathcal{L}(X)$. Then there exist constants $K, M>0$ such that for $s \in \rho(A)$ with $d(s, \sigma(A))>M$ we have $s \in \rho(A+P)$ and

$$
\left\|(s-(A+P))^{-1}\right\| \leq \frac{K}{d(s, \sigma(A))} .
$$

Proof. By Lemma 3.2 there exists $M_{1}, C>0$ such that

$$
\left\|(s-A)^{-1}\right\| \leq \frac{C}{d(s, \sigma(A))},
$$

for $s \in \rho(A)$ with $d(s, \sigma(A))>M_{1}$. Set $M:=\max \left\{M_{1}, 2\|P\| C\right\}$. Let $s \in \rho(A)$ with $d(s, \sigma(A))>M$. Then $I-P(s-A)^{-1}$ is invertible and we obtain

$$
\begin{aligned}
\left\|(s-(A+P))^{-1}\right\| & =\left\|(s-A)^{-1}\left[I-P(s-A)^{-1}\right]^{-1}\right\| \\
& \leq \frac{C}{d(s, \sigma(A))} \frac{1}{1-\|P\|\left\|(s-A)^{-1}\right\|} \\
& \leq \frac{2 C}{d(s, \sigma(A))} .
\end{aligned}
$$

Proposition 3.4. DS71 Let $A$ be an operator with $\sigma(A)=\left(s_{n}\right)_{n \in \mathbb{N}}$ such that the family of spectral subspaces is a Riesz basis of subspaces of $X$. Then $A$ has the representation $A=S+N$, where the scalar part $S$ is defined as

$$
\begin{aligned}
S x & :=\sum_{n \in \mathbb{N}} s_{n} E_{n} x, \\
\mathcal{D}(S) & =\left\{x \in X \mid \sum_{n=1}^{\infty}\left\|s_{n} E_{n} x\right\|^{2}<\infty\right\},
\end{aligned}
$$

and $N_{n}:=N E_{n}=\left(A-s_{n}\right) E_{n}$. Furthermore, $N_{n}$ is quasi-nilpotent, i.e., $\sigma\left(N_{n}\right)=\{0\}$ for all $n \in \mathbb{N}$. 
Proposition 3.5. Let $A$ be a generator of $C_{0}$-group on $X$ with compact resolvent. The eigenvalues are counted with algebraic multiplicity. If the following conditions are both fulfilled,

I. The span of the (generalized) eigenvectors form a dense set in $X$;

II. The eigenvalues can be decomposed into finitely many sets each having a uniform gap;

then $A$ is a discrete Riesz spectral operator.

Proof. By [Ww10, Theorem 1.1 and Theorem 1.6] it follows that the family of spectral subspaces is a Riesz basis of subspaces of $X$.

Thus, $A$ has the representation $A=S+N$, c.f. Proposition 3.4 where $S$ denotes the scalar part of the spectral operator $A$ and $N:=\sum_{n \in \mathbb{N}} N_{n}$, where $N_{n}=\left(A-s_{n}\right) E_{n}$ and $N_{n}$ is quasi-nilpotent. To prove that $A$ is a discrete Riesz spectral operator it remains to prove hat $N$ is bounded and nilpotent.

We can identify $N_{n}$ with a square matrix corresponding to $\left.N_{n}\right|_{E_{n} X}$ and thus $N_{n}$ is bounded and nilpotent.

Since the eigenvalues $A$ can be decomposed into finitely many sets each having a uniform gap, $N$ is nilpotent.

Finally, we verify that $N$ is bounded. Without loss of generality we assume that $A$ generates an exponentially stable $C_{0}$-group. Then by [W63, there exists an invertible and positive operator $L \in \mathcal{L}(X)$ such that

$$
\langle A x, L x\rangle+\langle x, L A x\rangle=-\langle x, x\rangle \quad \forall x \in \mathcal{D}(A) .
$$

We define $A_{n}:=A E_{n}=\left(s_{n}+N_{n}\right) E_{n}$, where $E_{n}$ denotes the $n$th spectral projection, and we identify $A_{n}$ and $N_{n}$ with the corresponding matrices on $E_{n} X$. From now on we fix $n$. Then we get for $x \in X$

$$
\left\langle A E_{n} x, L E_{n} x\right\rangle+\left\langle E_{n} x, L A E_{n} x\right\rangle=-\left\langle E_{n} x, E_{n} x\right\rangle
$$

or equivalently

$$
\left\langle A_{n} E_{n} x, L_{n} E_{n} x\right\rangle+\left\langle E_{n} x, L_{n} A_{n} E_{n} x\right\rangle=-\left\langle E_{n} x, E_{n} x\right\rangle,
$$

where $L_{n}:=E_{n}^{*} L E_{n}=L_{n}^{*}$. As $L$ is self-adjoint, $L_{n}$ is self-adjoint as well. Again we identify $L_{n}$ with the corresponding matrix on $E_{n} X$ and obtain on $E_{n} X$

$$
\left(s_{n}+N_{n}\right)^{*} L_{n}+L_{n}\left(s_{n}+N_{n}\right)=-I .
$$

Thus we have

$$
N_{n}^{*} L_{n}+L_{n} N_{n}=-I+r_{n} L_{n}
$$

with $r_{n}:=-2 \operatorname{Re} s_{n}$. Multiplying (77) from the right by $N_{n}^{k_{n}-j+1}$ and from the left by $\left(N_{n}^{*}\right)^{k_{n}-j}$ with $j=2,3, \ldots, k_{n}$ gives

$$
\begin{aligned}
\left(N_{i}^{*}\right)^{k_{n}-j+1} L_{n} N_{n}^{k_{n}-j+1}+ & \left(N_{n}^{*}\right)^{k_{n}-j} L_{n} N_{n}^{k_{n}-j+2} \\
& =-\left(N_{n}^{*}\right)^{k_{n}-j} N_{n}^{k_{n}-j+1}+r_{n}\left(N_{n}^{*}\right)^{k_{n}-j} L_{n} N_{n}^{k_{n}-j+1}
\end{aligned}
$$


and thus it holds

$$
\begin{aligned}
\left\|L_{n}^{1 / 2} N_{n}^{k_{n}-j+1}\right\|^{2} \leq & \left\|N_{n}^{k_{n}-j}\right\|\left\|N_{n}^{k_{n}-j+1}\right\|+\left|r_{n}\right|\left\|L_{n}\right\|\left\|N_{n}^{k_{n}-j}\right\|\left\|N_{n}^{k_{n}-j+1}\right\| \\
& +\left\|N_{n}^{k_{n}-j}\right\|\left\|L_{n}\right\|\left\|N_{n}^{k_{n}-j+2}\right\| .
\end{aligned}
$$

Since $L_{n}$ is boundedly invertible on $E_{n} X$, we get

$$
m\left\|N_{n}^{k_{n}-j+1}\right\|^{2} \leq\left\|L_{n}^{1 / 2} N_{n}^{k_{n}-j+1}\right\|^{2} \text { for some } m \text { independent of } n .
$$

For $j=2$ we use $N_{n}^{k_{n}}=0$ and obtain

$$
m\left\|N_{n}^{k_{n}-1}\right\| \leq\left\|N_{n}^{k_{n}-2}\right\|+\left|r_{n}\right|\left\|L_{n}\right\|\left\|N_{n}^{k_{n}-2}\right\| .
$$

Since $A$ is the generator of a $C_{0}$-group, we have $R:=\sup _{n \in \mathbb{N}}\left|r_{n}\right|<\infty$. Moreover, with $M:=\sup _{n \in \mathbb{N}}\left\|L_{n}\right\|<\infty$ and $C:=1+R M$, this implies

$$
\left\|N_{n}^{k_{n}-1}\right\| \leq \frac{C}{m}\left\|N_{n}^{k_{n}-2}\right\| .
$$

For $j=3, \ldots, k_{n}$ we get using (9), (8), and (10) and by induction over $j$

$$
\left\|N_{n}^{k_{n}-j+1}\right\| \leq\left\|N_{n}^{k_{n}-j}\right\| \sum_{l=1}^{j-1} \frac{C}{m^{l}} M^{l-1} .
$$

In particular, for $j=k_{n}$ and using $k_{n} \leq K$, this implies

$$
\left\|N_{n}\right\| \leq \sum_{l=1}^{k_{n}-1} \frac{C}{m^{l}} M^{l-1} \leq \sum_{l=1}^{K-1} \frac{C}{m^{l}} M^{l-1} .
$$

Together with Proposition 3.1 this implies that $N$ is bounded.

\section{Review on port-Hamiltonian systems}

The proof of our main result Theorem 2.8 requires some methods and results from port-Hamiltonian control systems. We define the (maximal) portHamiltonian operator by

$$
\mathfrak{A} x:=\left(P_{1} \frac{d}{d \zeta}+P_{0}\right)(\mathcal{H} x), \quad x \in \mathcal{D}(\mathfrak{A}),
$$

on $X:=L^{2}\left((0,1) ; \mathbb{C}^{d}\right)$ with the domain $\mathcal{D}(\mathfrak{A}):=\left\{x \in X \mid \mathcal{H} x \in H^{1}\left((0,1) ; \mathbb{C}^{d}\right)\right\}$. We equip the linear port-Hamiltonian system 1 with a boundary control $u$, that is, we consider

$$
\begin{aligned}
\frac{\partial x}{\partial t}(\zeta, t) & =\left(P_{1} \frac{\partial}{\partial \zeta}+P_{0}\right)(\mathcal{H}(\zeta) x(\zeta, t)), \\
x(\zeta, 0) & =x_{0}(\zeta), \\
u(t) & =\left[\begin{array}{ll}
W_{1} & W_{0}
\end{array}\right]\left[\begin{array}{l}
(\mathcal{H} x)(1, t) \\
(\mathcal{H} x)(0, t)
\end{array}\right],
\end{aligned}
$$

where $\zeta \in[0,1]$ and $t \geq 0$ and the Assumption 2.6 is fulfilled. 
Definition 4.1. We call the port-Hamiltonian system (12) a well-posed control system if $A$ generates a $C_{0}$-semigroup on $X$ and there exist $\tau>0$ and $m_{\tau} \geq 0$ such that for all $x_{0} \in \mathcal{D}(\mathfrak{A})$ and $u \in C^{2}\left([0, \tau] ; \mathbb{C}^{d}\right)$ with $u(0)=\left[\begin{array}{l}\left(\mathcal{H} x_{0}\right)(1,0) \\ \left(\mathcal{H} x_{0}\right)(0,0)\end{array}\right]$ the classical solution $x$ of (12) satisfies

$$
\|x(\tau)\|_{X}^{2} \leq m_{\tau}\left(\left\|x_{0}\right\|_{X}^{2}+\int_{0}^{\tau}\|u(t)\|^{2} d t\right) .
$$

There exists a rich literature on well-posed control systems, see e.g. Staffans Sta05 and Tuscnak and Weiss TW09. In general, it is not easy to show that a control system is well-posed. However, for the port-Hamiltonian control system (12) well-posedness is already satisfied if $A$ generates a $C_{0}$-semigroup, c.f. [ZLMV10, Theorem 3.3] and [JZ12, Theorem 13.2.2].

In the following, we assume that the port-Hamiltonian system (12) is a wellposed control system.

Well-posedness implies that for every initial condition $x_{0} \in X$ and every $L^{2}$ control function $u$ the port-Hamiltonian control system has a unique mild solution.

In order to define the mild solution we need to introduce some notation. Let $X_{-1}$ be the completion of $X$ with respect to the norm $\|x\|_{X_{-1}}=\left\|(\beta-A)^{-1} x\right\|_{X}$ for some $\beta$ in the resolvent set $\rho(A)$ of $A$. This implies, $X \subset X_{-1}$ and $X$ is continuously embedded and dense in $X_{-1}$. Moreover, let $(T(t))_{t \geq 0}$ be the $C_{0}$-semigroup generated by $A$ with growth bound $\omega_{0}(T)$. The $C_{0}$-semigroup $(T(t))_{t \geq 0}$ extends uniquely to a $C_{0}$-semigroup $\left(T_{-1}(t)\right)_{t \geq 0}$ on $X_{-1}$ whose generator $A_{-1}$, with domain equal to $X$, is an extension of $A$, see e.g. EN00.

Well-posedness implies the existence of $\tilde{B} \in \mathcal{L}\left(\mathbb{C}^{d}, X\right)$ with $\operatorname{ran} \tilde{B} \subset \mathcal{D}(\mathfrak{A})$ and $\mathfrak{A} \tilde{B} \in \mathcal{L}\left(\mathbb{C}^{d}, X\right)$ such that the unique mild solution of (12) can be defined by

$$
x(t)=T(t) x_{0}+\int_{0}^{t} T_{-1}(t-s)\left(\mathfrak{A} \widetilde{B}-A_{-1} \widetilde{B}\right) u(s) d s,
$$

see [JZ18].

The port-Hamiltonian control system can be

written equivalently in the standard control operator formulation

$$
\dot{x}(t)=A_{-1} x(t)+B u(t) \quad x(0)=x_{0}, \quad t \geq 0,
$$

where $B \in \mathcal{L}\left(\mathbb{C}^{d}, X_{-1}\right)$ given by

$$
B:=\mathfrak{A} \widetilde{B}-A_{-1} \widetilde{B}
$$

The operator $B$ is an admissible control operator for $(T(t))_{t \geq 0}$, that is,

$$
\Phi_{t_{f}} u:=\int_{0}^{t_{f}} T_{-1}\left(t_{f}-s\right) B u(s) d s \in X
$$

for every $u \in L^{2}\left(\left(0, t_{f}\right) ; \mathbb{C}^{d}\right)$. Admissibility implies that the mild solution of (12) satisfies $x \in C\left(\left[0, t_{f}\right) ; X\right)$ for every initial condition $x_{0} \in X$ and every $u \in L^{2}\left(\left(0, t_{f}\right) ; \mathbb{C}^{d}\right)$. 
Well-posed port-Hamiltonian control systems are always exactly controllable in finite time as the following proposition shows.

Proposition 4.2. JJK19a] Every well-posed port-Hamiltonian control system (12) is exactly controllable in finite time, that is, there exists a time $\tau>0$ such that for all $x_{1} \in X$ there exists a control function $u \in L^{2}\left((0, \tau) ; \mathbb{C}^{d}\right)$ such that the corresponding mild solution satisfies $x(0)=0$ and $x(\tau)=x_{1}$.

As a consequence of exact controllability we obtain that the eigenspaces span the state space.

Proposition 4.3. Consider a well-posed port-Hamiltonian control system (12) and assume that $A$ generates a $C_{0}$-group on $X$. Let $\sigma(A)=\left(s_{n}\right)_{n \in \mathbb{N}}$. Then

$$
X=\overline{\operatorname{span}_{n \in \mathbb{N}} E\left(\left(s_{n}\right)\right) X},
$$

where $E\left(\left(s_{n}\right)\right)$ is introduced in Definition 2.1.

Proof. Follows from [JZ99, Lemma 7.3] together with Proposition 4.2 .

\section{Proof of the Main Result}

This section is devoted to the proof of Theorem 2.8. Let us first assume that one of the conditions of the theorem is satisfied. As the resolvent of $A$ is compact the spectrum consists of isolated eigenvalues only. That the eigenvalues lie in a strip parallel to the imaginary axis and that they can be decomposed into finitely many sets each having a uniform gap will be shown in the proof of the implication 2) $\Rightarrow 1$ ). Finally, $\omega_{0}(A)=s(A)$ is implied by [GZ01, Theorem 2.12].

\subsection{Proof of the equivalence 2) $\Leftrightarrow 3$ ) of Theorem 2.8}

The operator $A$ generates a $C_{0^{-}}$group, if and only if $A$ and $-A$ generates a $C_{0^{-}}$ semigroup, [EN00, Section II.3.11]. In [JMZ15, Theorem 1.5] shows that $A$ is the generator of a $C_{0}$-semigroup if and only if $W_{1} \mathcal{H}(1) Z^{+}(1) \oplus W_{0} \mathcal{H}(0) Z^{-}(0)=\mathbb{C}^{d}$. Since $\mathcal{D}(-A)=\mathcal{D}(A)$ and $\sigma_{p}\left(P_{1} \mathcal{H}\right)=-\sigma_{p}\left(-P_{1} \mathcal{H}\right)$ it follows that $-A$ generates a $C_{0}$-semigroup if and only if $W_{1} \mathcal{H}(1) Z^{-}(1) \oplus W_{0} \mathcal{H}(0) Z^{+}(0)=\mathbb{C}^{d}$.

\subsection{Proof of the implication 2) $\Rightarrow$ 1) of Theorem 2.8}

The following lemma will be useful.

Lemma 5.1. Let $s \in \mathbb{C}$ and $P_{1}, P_{0}$ and $\mathcal{H}$ fulfil the condition for a port-Hamiltonian operator in Assumption 2.6. Then the solutions of the system of ordinary differential equations

$$
s x(\zeta)=\left(P_{1} \frac{d}{d \zeta}+P_{0}\right)(\mathcal{H} x)(\zeta), \quad \zeta \in[0,1],
$$


denoted by $x(\zeta)=\Psi^{s}(\zeta) x(0)$, satisfy

$$
\tilde{M} e^{-|\operatorname{Re} s| \tilde{c}_{0} \zeta}\|v\| \leq\left\|\Psi^{s}(\zeta) v\right\| \leq M e^{|\operatorname{Re} s| c_{0} \zeta}\|v\|, \quad v \in \mathbb{C}^{d}, \zeta \in[0,1],
$$

with constants $M, \tilde{M}>0$, and $\tilde{c}_{0}, c_{0} \geq 0$ independent of $s$ and $\zeta$.

Proof. Writing $\tilde{x}=\mathcal{H} x$, (15) can be equivalently written as

$$
\mathcal{H}(\zeta) P_{1} \tilde{x}^{\prime}(\zeta)=s \tilde{x}(\zeta)-\mathcal{H}(\zeta) P_{0} \tilde{x}(\zeta)
$$

We write $s=i \omega+r$ with $\omega, \tau \in \mathbb{R}$ and diagonalize $P_{1} \mathcal{H}(\zeta)=S^{-1}(\zeta) \Delta(\zeta) S(\zeta)$, see Assumption 2.6. Thus, $\mathcal{H}(\zeta) P_{1}=S^{*}(\zeta) \Delta(\zeta) S^{-*}(\zeta)$ and we get

$$
\Delta(\zeta) S^{-*}(\zeta) \tilde{x}^{\prime}(\zeta)=i \omega S^{-*}(\zeta) \tilde{x}(\zeta)+\left(r I-S^{-*}(\zeta) \mathcal{H}(\zeta) P_{0} S^{*}(\zeta)\right) S^{-*}(\zeta) \tilde{x}(\zeta) .
$$

Using the substitution $z=S^{-*} \tilde{x}$ gives the equivalent differential equation

$$
z^{\prime}(\zeta)=i \omega \Delta^{-1}(\zeta) z(\zeta)+\left(r \Delta^{-1}(\zeta)+Q(\zeta)\right) z(\zeta),
$$

where

$$
Q(\zeta):=-\Delta^{-1}(\zeta) S^{-*}(\zeta) \mathcal{H}(\zeta) P_{0} S^{*}(\zeta)-\left(S^{-*}\right)^{\prime}(\zeta) S^{*}(\zeta)
$$

Thus, equation (15) is equivalent to equation (16). Due to the fact that $P_{1} \mathcal{H}(\zeta)$ has real eigenvalues, $\Delta(\zeta)$ is a diagonal, real matrix and $i \omega \Delta^{-1}(\zeta)$ is a diagonal, purely imaginary matrix. We write $\Delta^{-1}(\zeta)=\operatorname{diag}_{k=1, \ldots, n}\left(\alpha_{k}(\zeta)\right)$ with $\alpha_{k}(\zeta)$ : $[0,1] \rightarrow \mathbb{R}$ and define $\Phi_{\omega}(\zeta)=\operatorname{diag}\left(\exp \left(-i \omega \int_{0}^{\zeta} \alpha_{k}(\tau) d \tau\right)\right)$ which satisfies

$$
\left\|\Phi_{\omega}(\zeta)\right\|_{\mathcal{L}\left(\mathbb{C}^{d}\right)}=1, \quad \zeta \in[0,1] .
$$

Multiplying (16) with $\Phi_{\omega}(\zeta)$, we get

$$
\Phi_{\omega}(\zeta) z^{\prime}(\zeta)-i \omega \Delta^{-1}(\zeta) \Phi_{\omega}(\zeta) z(\zeta)=\Phi_{\omega}(\zeta)\left(r \Delta^{-1}(\zeta)+Q(\zeta)\right) z(\zeta)
$$

or equivalently

$$
\left(\Phi_{\omega}(\zeta) z(\zeta)\right)^{\prime}=\left(r \Delta^{-1}(\zeta)+\Phi_{\omega}(\zeta) Q(\zeta) \Phi_{\omega}^{-1}(\zeta)\right) \Phi_{\omega}(\zeta) z(\zeta) .
$$

Using the substitution $y=\Phi_{\omega} z$, this ordinary differential equation becomes

$$
y^{\prime}=\left(r \Delta^{-1}(\zeta)+Q_{\omega}(\zeta)\right) y(\zeta),
$$

where $Q_{\omega}(\zeta):=\Phi_{\omega}(\zeta) Q(\zeta) \Phi_{\omega}^{-1}(\zeta)$. There exist constants $c_{0}, c_{1} \geq 0$, independent of $\omega$, such that

$$
2 \max _{\zeta \in[0,1]}\left\|r \Delta^{-1}(\zeta)+Q_{\omega}(\zeta)\right\| \leq|r| c_{0}+c_{1}
$$

The solution $y$ of (17) satisfies

$$
\frac{d}{d \zeta}\|y(\zeta)\|^{2}=y(\zeta)^{*}\left[\left(r \Delta^{-1}(\zeta)+Q_{\omega}(\zeta)\right)+\left(r \Delta^{-1}(\zeta)+Q_{\omega}(\zeta)\right)^{*}\right] y(\zeta)
$$


This together with (18) implies

$$
-\left(|r| c_{0}+c_{1}\right)\|y(\zeta)\|^{2} \leq \frac{d}{d \zeta}\|y(\zeta)\|^{2} \leq\left(|r| c_{0}+c_{1}\right)\|y(\zeta)\|^{2},
$$

and thus

$$
e^{-\left(|r| c_{0}+c_{1}\right) \zeta}\|y(0)\|^{2} \leq\|y(\zeta)\|^{2} \leq e^{\left(|r| c_{0}+c_{1}\right) \zeta}\|y(0)\|^{2} .
$$

As the mapping $x \mapsto y$ is boundedly invertible on $L^{2}\left((0,1), \mathbb{C}^{d}\right)$, with norm independent on $\omega$, the statement follows.

Next we state some results from complex analysis.

Definition 5.2. AI95, II.1.27] An entire function $f$ is called an entire function of exponential type, if there exist constants $C$ and $T$ such that $|f(s)| \leq C e^{T|s|}$ for all $s \in \mathbb{C}$. Further, an entire function $f$ of exponential type is said to be of sine type, if

1. the zeros of $f$ lie in a strip $\{s \in \mathbb{C}|| \operatorname{Im} s \mid \leq h\}$ for some $h \geq 0$.

2. there exist $\hat{\omega} \in \mathbb{R}$ and positive constants $c$ and $C$ such that $c \leq|f(r+i \hat{\omega})| \leq$ $C$ for every $r \in \mathbb{R}$ holds.

Proposition 5.3. [AI95, Proposition II.1.28] (Levin 1961) If $f$ is of sine type, then its set of zeros counted with algebraic multiplicity is a finite unification of sets each having a uniform gap.

Lemma 5.4. A complex number $s \in \mathbb{C}$ is an eigenvalue of a port-Hamiltonian operator $A$ if and only if

$$
\operatorname{det}\left[W_{1} \mathcal{H}(1) \Psi^{s}(1)+W_{0} \mathcal{H}(0)\right]=0,
$$

where $\Psi^{s}$ is described in Lemma 5.1.

Proof. For every $x(0) \in \mathbb{C}^{d}$ there exists a solution of the differential equation $s x(\zeta)=\left(P_{1} \frac{d}{d \zeta}+P_{0}\right)(\mathcal{H} x)(\zeta), \zeta \in[0,1]$. The complex number $s$ is an eigenvalue of $A$ if and only if $x \in \mathcal{D}(A)$ and $A x=s x$. Using Lemma 5.1 this is equivalent to

$$
x(\zeta)=\Psi^{s}(\zeta) x(0) \text { and }\left[\begin{array}{ll}
W_{1} & W_{0}
\end{array}\right]\left[\begin{array}{l}
(\mathcal{H} x)(1) \\
(\mathcal{H} x)(0)
\end{array}\right]=0 .
$$

Inserting the first equation in the second, we get that $s$ is an eigenvalue of $A$ if and only if $\operatorname{det}\left[W_{1} \mathcal{H}(1) \Psi^{s}(1)+W_{0} \mathcal{H}(0)\right]=0$.

Now we are in the situation to give the proof of the implication 2 ) $\Rightarrow 1$ ). 
Proof of the implication 2) $\Rightarrow$ 1) of Theorem 2.8. Assertion 2) implies that the eigenvalues lie in a strip parallel to the imaginary axis. Thus, thanks to Proposition 4.3 and Proposition 3.5 it suffices to show that the eigenvalues $\left(s_{n}\right)_{n \in \mathbb{N}}$ of $A$ (counted according to the algebraic multiplicity) can decomposed into finitely many sets having each a uniform gap.

Using Proposition 5.3, this is implied by the existence of an entire function $g$ of exponential type with

i) $g$ has exactly the zeros $\left(s_{n}\right)_{n \in \mathbb{N}}$ and

ii) there exist $r, c, C>0$ such that for every $\omega \in \mathbb{R}: c \leq|g(r+i \omega)| \leq C$.

Note that $f: \mathbb{C} \rightarrow \mathbb{C}$, defined by $f(s):=g(i s)$ is a sine-type function if $g$ is an entire function of exponential type and satisfying the above conditions $i$ ) and ii). We define $g: \mathbb{C} \rightarrow \mathbb{C}$ by

$$
g(s):=\operatorname{det}\left[W_{1} \mathcal{H}(1) \Psi^{s}(1)+W_{0} \mathcal{H}(0)\right]
$$

By Lemma 5.4, a complex number $s \in \mathbb{C}$ is an eigenvalue of the operator $A$ if and only if $g(s)=0$.

$\Psi^{s}$ described in Lemma 5.1 is an entire function, c.f. Was87, Theorem 24.1] and thus $g$ is an entire function as well. Clearly, $g$ has the zeros $\left(s_{n}\right)_{n \in \mathbb{N}}$. Since the determinant of a matrix equals the product of its eigenvalues and every eigenvalue is smaller or equal the norm of the matrix, it yields $|g(s)| \leq$ $\left\|\left[W_{1} \mathcal{H}(1) \Psi^{s}(1)+W_{0} \mathcal{H}(0)\right]\right\|^{n}$. Using Lemma [5.1] it holds $|g(s)| \leq c_{2} e^{|\operatorname{Re} s| c_{3}}$ for some constants $c_{2}, c_{3} \geq 0$, and thus, $g$ is bounded on lines parallel to the imaginary axis and grows at most exponentially.

Next, we show that $g$ is bounded away from zero on some line parallel to the imaginary axis. Since the control operator $B$ of the port-Hamiltonian system (12) is admissible, see Section 4, it yields that for $\omega>\omega_{0}(A)$ exists a constant $M_{\omega}>0$ such that

$$
\left\|\left(s-A_{-1}\right)^{-1} B\right\|_{\mathcal{L}\left(\mathbb{C}^{d}, X\right)} \leq \frac{M_{\omega}}{\sqrt{\operatorname{Re} s-\omega}} \quad \text { for } \operatorname{Re} s \geq \omega,
$$

see [TW14, Proposition 4.4.6]. Let $r>\omega_{0}(A)$ and we assume that $g$ is not bounded away from zero on $r+i \mathbb{R}$, i.e., there exists a sequence $\omega_{k} \in \mathbb{R}$ such that $g\left(r+i \omega_{k}\right) \rightarrow 0$. Since all zeros of $g$ have real part less or equal to the growth bound $\omega_{0}(T)$ of the $C_{0}$-semigroup generated by $A$, it holds true that $g\left(r+i \omega_{k}\right) \neq 0$. Let $u_{0}$ be an arbitrary vector in $\mathbb{C}^{d}$. By [JZ12, Theorem 12.1.3] the solution $x_{u_{0}}^{r+i \omega_{k}}$ of

$$
\begin{array}{r}
\left(r+i \omega_{k}\right) x(\zeta)=\left(P_{1} \frac{d}{d \zeta}+P_{0}\right)(\mathcal{H} x)(\zeta) \\
{\left[\begin{array}{ll}
W_{1} & W_{0}
\end{array}\right]\left[\begin{array}{l}
(\mathcal{H} x)(1) \\
(\mathcal{H} x)(0)
\end{array}\right]=u_{0},}
\end{array}
$$


is given by

$$
\begin{aligned}
x_{u_{0}}^{r+i \omega_{k}} & =\left(\left(\left(r+i \omega_{k}\right)-A_{-1}\right)^{-1}\left(\mathfrak{A} \tilde{B}-\left(r+i \omega_{k}\right) \tilde{B}\right)+\tilde{B}\right) u_{0} \\
& =\left(\left(r+i \omega_{k}\right)-A_{-1}\right)^{-1} B u_{0}
\end{aligned}
$$

and $x_{u_{0}}^{r+i \omega_{k}}(0)$ fulfils

$$
\left[W_{1} \mathcal{H}(1) \Psi^{r+i \omega_{k}}(1)+W_{0} \mathcal{H}(0)\right] x_{u_{0}}^{r+i \omega_{k}}(0)=u_{0} .
$$

Let $M_{k}:=\left[W_{1} \mathcal{H}(1) \Psi^{r+i \omega_{k}}(1)+W_{0} \mathcal{H}(0)\right]$. Since $g\left(r+i \omega_{k}\right)=\operatorname{det}\left(M_{k}\right) \rightarrow 0$ and $1=\operatorname{det}(I)=\operatorname{det}\left(M_{k}\right) \operatorname{det}\left(M_{k}^{-1}\right)$, we have $\operatorname{det}\left(M_{k}^{-1}\right) \rightarrow \infty$. Hence, $M_{k}^{-1}$ has an eigenvalue $\nu_{k}$ with $\left|\nu_{k}\right| \rightarrow \infty$. Choose $u_{k, 0}$ as a normalized eigenvector to $\nu_{k}$. Then it yields

$$
x_{u_{k, 0}}^{r+i \omega_{k}}(0)=M_{k}^{-1} u_{k, 0}=\nu_{k} u_{k, 0},
$$

which implies $\left\|x_{u_{k, 0}}^{r+i \omega_{k}}(0)\right\|_{\mathbb{C}^{d}} \rightarrow \infty$. We note that the function $x_{u_{k, 0}}^{r+i \omega_{k}}$ is given by

$$
x_{u_{k, 0}}^{r+i \omega_{k}}(\zeta)=\Psi^{r+i \omega_{k}}(\zeta) x_{u_{k, 0}}^{r+i \omega_{k}}(0) .
$$

Using that the inverse of $\Psi^{r+i \omega_{k}}$ is a bounded function, see Lemma 5.1, we get

$$
\left\|x_{u_{k, 0}}^{r+i \omega_{k}}\right\|_{L^{2}\left((0,1) ; \mathbb{C}^{d}\right)} \rightarrow \infty .
$$

However, since we also have $x_{u_{k, 0}}^{r+i \omega_{k}}=\left(\left(r+i \omega_{k}\right)-A_{-1}\right)^{-1} B u_{k, 0}$, equation (22) is in contradiction with the uniform boundedness of $\left(\left(r+i \omega_{k}\right)-A_{-1}\right)^{-1} B u_{0, k}$, see equation (21). Thus the entire function $g$ is of exponential type and satisfies condition $i$ ) and $i i$ ). This concludes the proof.

\subsection{Proof of the implication 1) $\Rightarrow 2$ ) of Theorem 2.8}

We start with some characterizations of the resolvent and the spectrum of portHamiltonian operators.

Lemma 5.5. Let $\Lambda \in C\left([0,1] ; \mathbb{C}^{d}\right)$ with $\Lambda(\zeta)$ diagonal, invertible und positive definite for every $\zeta \in[0,1], Q \in \mathbb{C}^{n \times n}$ singular and $\left.A: \mathcal{D}(A) \subset L^{2}((0,1)) ; \mathbb{C}^{d}\right) \rightarrow$ $\left.L^{2}((0,1)) ; \mathbb{C}^{d}\right)$ defined by $A x=\Lambda x^{\prime}$ and $\mathcal{D}(A)=\left\{x \in H^{1}\left((0,1) ; \mathbb{C}^{d}\right) \mid x(1)+\right.$ $Q x(0)=0\}$. Then there exist real constants $\gamma<0$ and $a, b>0$ such that $\left\|(s-A)^{-1}\right\| \geq a e^{b|s|}$ for real $s \in \rho(A)$ with $s<\gamma$.

Proof. Let $0 \neq x(0) \in \operatorname{ker} Q$. We define $F_{s}(\zeta):=s \int_{0}^{\zeta} \Lambda^{-1}(\tau) d \tau$ for $s \in \mathbb{R}$ and $\zeta \in[0,1]$. Note that $F_{s}$ and $\Lambda$ commute as both are diagonal. There exists $\gamma_{0}<0$ such that $I-e^{2 F_{s}(1)}$ is invertible if $s<\gamma_{0}$. Further, let $s \in \rho(A)$ with $s<\gamma_{0}$ and define

$$
g(\zeta)=2 s e^{F_{s}(1)-F_{s}(\zeta)} g_{0}=2 s e^{s \int_{\zeta}^{1} \Lambda^{-1}(\tau) d \tau} g_{0}
$$


with $g_{0}:=e^{F_{s}(1)}\left(I-e^{2 F_{s}(1)}\right)^{-1} x(0)$. Then the solution of

$$
\Lambda x^{\prime}=s x+g
$$

is given by

$$
\begin{aligned}
x(\zeta) & =e^{F_{s}(\zeta)} x(0)+\int_{0}^{\zeta} e^{F_{s}(\zeta)-F_{s}(\tau)} \Lambda^{-1}(\tau) g(\tau) d \tau \\
& =e^{F_{s}(\zeta)} x(0)-e^{F_{s}(\zeta)+F_{s}(1)} \int_{0}^{\zeta}\left(-2 s \Lambda^{-1}(\tau)\right) e^{-2 F_{s}(\tau)} d \tau g_{0} \\
& =e^{F_{s}(\zeta)} x(0)-e^{F_{s}(\zeta)+F_{s}(1)}\left(e^{-2 F_{s}(\zeta)}-I\right) g_{0} \\
& =e^{F_{s}(\zeta)}\left(I-e^{2 F_{s}(1)}\right) e^{-F_{s}(\zeta)} g_{0}-e^{-F_{s}(\zeta)+F_{s}(1)} g_{0}+e^{-F_{s}(\zeta)+F_{s}(1)} g_{0} \\
& =e^{F_{s}(\zeta)-F_{s}(1)} g_{0}-\frac{1}{2 s} g(\zeta) .
\end{aligned}
$$

In particular $x(1)=0$ and thus $(s-A)^{-1} g=x$. As $\Lambda \in C\left([0,1] ; \mathbb{C}^{d}\right)$ is a diagonal and invertible matrix-valued function with positive entries on its diagonal, there exists $\lambda_{0}>0$ such that the diagonal elements of $\Lambda$ are bounded by $\lambda_{0}$. First using (23) we can estimate

$$
\|g\|_{L^{2}\left((0,1) ; \mathbb{C}^{d}\right)} \leq c \sqrt{|s|}\left\|g_{0}\right\|, c>0
$$

and

$$
\begin{aligned}
\|x\|_{L^{2}\left(0,1 ; \mathbb{C}^{d}\right)} & \geq\left\|e^{F_{s}(\cdot)-F_{s}(1)} g_{0}\right\|_{L^{2}\left(0,1 ; \mathbb{C}^{d}\right)}-\frac{1}{2|s|}\|g\|_{L^{2}\left((0,1) ; \mathbb{C}^{d}\right)} \\
& =\left(\int_{0}^{1}\left\|e^{-s \int_{\tau}^{1} \Lambda^{-1}(\sigma) d \sigma} g_{0}\right\|^{2} d \tau\right)^{\frac{1}{2}}-\frac{1}{2|s|}\|g\|_{L^{2}\left((0,1) ; \mathbb{C}^{d}\right)} \\
& \geq\left(\int_{0}^{1} e^{2|s|(1-\tau) \lambda_{0}}\left\|g_{0}\right\|^{2} d \tau\right)^{\frac{1}{2}}-\frac{1}{2|s|}\|g\|_{L^{2}\left((0,1) ; \mathbb{C}^{d}\right)} \\
& \geq \frac{1}{4|s|^{2} \lambda_{0}}\left(e^{2|s| \lambda_{0}}-1\right)\|g\|_{L^{2}\left((0,1) ; \mathbb{C}^{d}\right)}-\frac{1}{2|s|}\|g\|_{L^{2}\left((0,1) ; \mathbb{C}^{d}\right)} .
\end{aligned}
$$

This completes the lemma.

Lemma 5.6. Let $A$ be a port-Hamiltonian operator, which generates a $C_{0}$-semigroup. Furthermore, let $A$ be a discrete Riesz spectral operator and let $\left(s_{n}\right)_{n \in \mathbb{N}}$ denote its eigenvalues. Then there exists a constant $K>0$ such that for every $n \in \mathbb{N}$ within the ball $\left\{s \in \mathbb{C}|| s-\left.s_{n}|\leq K| \operatorname{Re} s_{n}\right|^{2}\right\}$ there lie at most $d$ eigenvalues.

Proof. Without lost of generality we assume that $A$ generates an exponentially stable $C_{0}$-semigroup. By Proposition 4.2 the corresponding port-Hamiltonian control system (12) with control operator $B$ is exactly controllable in finite 
time. Then the dual system, described by $A^{*}$ and $B^{*}$, is exactly observable and it yields due to the Hautus Test, c.f. [RW94], that there exists a positive constant $m$ such that

$$
\left\|\left(s-A^{*}\right) x\right\|^{2}+|\operatorname{Re} s|\left\|B^{*} x\right\|^{2} \geq m|\operatorname{Re} s|^{2}\|x\|^{2}, \quad \operatorname{Re} s<0, x \in \mathcal{D}\left(A^{*}\right) .
$$

No matter that there may exist generalized eigenvectors, we consider only eigenvectors corresponding to different eigenvalues of $A^{*}$. As $\sigma(A)=\overline{\sigma\left(A^{*}\right)}$, it suffices to prove the statement for $A^{*}$. Choose arbitrary $e_{1}, \ldots e_{d+1}$ normed eigenvectors of the operator $A^{*}$ to the eigenvalues $\lambda_{1}, \ldots, \lambda_{d+1}$ with $\lambda_{n} \neq \lambda_{m}$ for $n \neq m$ $\in 1, \ldots, d+1$.

Since $B^{*} \in \mathcal{L}\left(\mathcal{D}\left(A^{*}\right), \mathbb{C}^{d}\right)$ the $d+1$ vectors $B^{*} e_{n}$ are linearly dependent in $\mathbb{C}^{d}$, i.e, there exists scalars $a_{1}, \ldots, a_{d+1} \in \mathbb{C}$ with $\sum_{n=1}^{d+1}\left|a_{n}\right|^{2}=1$ such that

$$
a_{1} B^{*} e_{1}+\ldots a_{d+1} B^{*} e_{d+1}=0
$$

Consider $x=\sum_{n=1}^{d+1} a_{n} e_{n}$. Then $x \in \mathcal{D}\left(A^{*}\right)$ with $B^{*} x=0$, and

$$
\|x\|^{2}=\left\|\sum_{n=1}^{d+1} a_{n} e_{n}\right\| \geq m_{1}>0,
$$

by Proposition 3.1. It follows with the Hautus Test (25) at the point $s=\lambda_{d+1}$

$$
\begin{aligned}
m m_{1}\left|\operatorname{Re} \lambda_{d+1}\right|^{2} & \leq\left\|\left(\lambda_{d+1}-A^{*}\right) x\right\|^{2}=\left\|\sum_{n=1}^{d} a_{n}\left(\lambda_{d+1}-\lambda_{n}\right) e_{n}\right\|^{2} \\
& \leq m_{2} \sum_{n=1}^{d}\left|a_{n}\right|^{2}\left|\lambda_{d+1}-\lambda_{n}\right|^{2}
\end{aligned}
$$

Thus,

$$
\frac{d+1}{d+1} \frac{m m_{1}}{m_{2}}\left|\operatorname{Re} \lambda_{d+1}\right|^{2} \leq \sum_{n=1}^{d}\left|\lambda_{d+1}-\lambda_{n}\right|^{2} .
$$

Since the eigenvalues are arbitrary chosen, this implies that in the ball with radius $\frac{m m_{1}}{(d+1) m_{2}}\left|\operatorname{Re} \lambda_{d+1}\right|^{2}$ around $\lambda_{d+1}$ lies at most $d$ eigenvalues. Hence, the statement follows.

Now we are in the position to prove the implication 1) $\Rightarrow 2$ ).

Proof of the implication 1) $\Rightarrow 2$ ) of Theorem 2.8: We assume that $A$ does not generate a $C_{0}$-group.

Since $A$ is a port-Hamiltonian operator, we denote by $S$ the matrix-valued function such that $P_{1} \mathcal{H}(\zeta)=S^{-1}(\zeta) \Delta(\zeta) S(\zeta)$, see Assumption 2.6. Since the eigenvalues of $P_{1} \mathcal{H}(\zeta)$ and $\mathcal{H}(\zeta)^{\frac{1}{2}} P_{1} \mathcal{H}(\zeta)^{\frac{1}{2}}$ are the same, it follows by Sylvesters law of inertia that the number of positive and negative eigenvalues of $P_{1} \mathcal{H}(\zeta)$ equal those of $P_{1}$. Let $d_{1}$ denote the number of positive and $d_{2}=d-d_{1}$ the 
number of negative eigenvalues of $P_{1}$. Thus without loss of generality $\Delta$ can be written as $\Delta(\zeta)=\left[\begin{array}{cc}\Lambda(\zeta) & 0 \\ 0 & \Theta(\zeta)\end{array}\right]$, where $\Lambda(\zeta) \in \mathbb{C}^{d_{1} \times d_{1}}$ correspond to the positive eigenvalues and $\Theta(\zeta) \in \mathbb{C}^{d_{2} \times d_{2}}$ to the negative ones.

Let $\mathcal{S} \in \mathcal{L}(X)$ be the multiplication operator $(\mathcal{S} x)(\zeta):=S(\zeta) x(\zeta)$. By assumption $\mathcal{S}$ is invertible and we obtain

$$
\begin{aligned}
\mathcal{S} A \mathcal{S}^{-1} z(\zeta)= & \Delta(\zeta)(z(\zeta))^{\prime} \\
& +\Delta^{\prime}(\zeta) z(\zeta)+S(\zeta)\left(S^{-1}\right)^{\prime} \Delta(\zeta) z(\zeta)+S(\zeta) P_{0} \mathcal{H}(\zeta) S^{-1}(\zeta) z(\zeta) \\
\mathcal{D}\left(\mathcal{S} A \mathcal{S}^{-1}\right)= & \left\{z \in H^{1}\left((0,1) ; \mathbb{C}^{d}\right) \mid\left[\begin{array}{ll}
W_{1} & W_{0}
\end{array}\right]\left[\begin{array}{l}
\left(\mathcal{H} S^{-1} z\right)(1) \\
\left(\mathcal{H} S^{-1} z\right)(0)
\end{array}\right]=0\right\} .
\end{aligned}
$$

The operator $\mathcal{S} A \mathcal{S}^{-1}$ generates a $C_{0}$-semigroup, too. We split the variable $z(\zeta)=\left[\begin{array}{l}z^{+}(\zeta) \\ z^{-}(\zeta)\end{array}\right] \in \mathbb{C}^{d}$ with $z^{+}(\zeta) \in \mathbb{C}^{d_{1}}$ and $z^{-}(\zeta) \in \mathbb{C}^{d_{2}}$, and we define $W_{1} \mathcal{H}(1) S^{-1}(1)=:\left[\begin{array}{ll}V_{1} & V_{2}\end{array}\right]$ and $W_{0} \mathcal{H}(0) S^{-1}(0)=:\left[\begin{array}{ll}U_{1} & U_{2}\end{array}\right]$, where $U_{1}, V_{1} \in$ $\mathbb{C}^{d \times d_{1}}$ and $U_{2}, V_{2} \in \mathbb{C}^{d \times d_{2}}$. Then, $z \in \mathcal{D}\left(\mathcal{S} A \mathcal{S}^{-1}\right)$ if and only if $z \in H^{1}\left((0,1) ; \mathbb{C}^{d}\right)$ and

$$
\begin{aligned}
0 & =\left[\begin{array}{ll}
W_{1} & W_{0}
\end{array}\right]\left[\begin{array}{l}
\left(\mathcal{H} S^{-1} z\right)(1) \\
\left(\mathcal{H} S^{-1} z\right)(0)
\end{array}\right] \\
& =\left[\begin{array}{ll}
V_{1} & V_{2}
\end{array}\right]\left[\begin{array}{l}
z^{+}(1) \\
z^{-}(1)
\end{array}\right]+\left[\begin{array}{ll}
U_{1} & U_{2}
\end{array}\right]\left[\begin{array}{l}
z^{+}(0) \\
z^{-}(0)
\end{array}\right] \\
& =\left[\begin{array}{ll}
V_{1} & U_{2}
\end{array}\right]\left[\begin{array}{l}
z^{+}(1) \\
z^{-}(0)
\end{array}\right]+\left[\begin{array}{ll}
U_{1} & V_{2}
\end{array}\right]\left[\begin{array}{l}
z^{+}(0) \\
z^{-}(1)
\end{array}\right] \\
& =K\left[\begin{array}{l}
\Lambda(1) z^{+}(1) \\
\Theta(0) z^{-}(0)
\end{array}\right]+Q\left[\begin{array}{l}
\Lambda(0) z^{+}(0) \\
\Theta(1) z^{-}(1)
\end{array}\right]
\end{aligned}
$$

where $K:=\left[\begin{array}{ll}V_{1} & U_{2}\end{array}\right]\left[\begin{array}{cc}\Lambda(1)^{-1} & 0 \\ 0 & \Theta(0)^{-1}\end{array}\right]$ and $Q:=\left[\begin{array}{ll}U_{1} & V_{2}\end{array}\right]\left[\begin{array}{cc}\Lambda(0)^{-1} & 0 \\ 0 & \Theta(1)^{-1}\end{array}\right]$. Since $\mathcal{S} A \mathcal{S}^{-1}$ is the generator of a $C_{0}$-semigroup and this property is invariant under bounded perturbations, $K$ is invertible, see [JZ12, Theorem 13.3.1].

Let $\mathcal{T} \in \mathcal{L}(X)$ be defined by $\mathcal{T}\left[\begin{array}{c}z^{+}(\zeta) \\ z^{-}(\zeta)\end{array}\right]:=\left[\begin{array}{c}z^{+}(\zeta) \\ z^{-}(1-\zeta)\end{array}\right]$. Clearly, $\mathcal{T}$ is invertible. Then the operator $\mathcal{A}:=\mathcal{T} \mathcal{S} A \mathcal{S}^{-1} \mathcal{T}^{-1}$ on $X$ is given by

$$
\begin{aligned}
& \mathcal{A} z(\zeta)=\left[\begin{array}{cc}
\Lambda(\zeta) & 0 \\
0 & -\Theta(1-\zeta)
\end{array}\right] z^{\prime}(\zeta)+R(\zeta) z(\zeta) \\
& \mathcal{D}(\mathcal{A})=\left\{z \in H^{1}\left((0,1) ; \mathbb{C}^{d}\right) \mid K\left[\begin{array}{cc}
\Lambda(1) & 0 \\
0 & \Theta(0)
\end{array}\right] z(1)+Q\left[\begin{array}{cc}
\Lambda(1) & 0 \\
0 & \Theta(0)
\end{array}\right] z(0)=0\right\},
\end{aligned}
$$

where $z \mapsto R(\cdot) z(\cdot)$ is a bounded multiplication operator on $X$. Let $\tilde{K}:=$ $K\left[\begin{array}{cc}\Lambda(1) & 0 \\ 0 & \Theta(0)\end{array}\right]$ and $\tilde{Q}:=Q\left[\begin{array}{cc}\Lambda(1) & 0 \\ 0 & \Theta(0)\end{array}\right]$. By assumption, the matrix $\tilde{K}$ is invertible. As a bounded perturbation of a generator of $C_{0}$-group generates again a $C_{0^{-}}$ 
group, we obtain that the operator

$$
\begin{aligned}
& \tilde{A} z(\zeta)=\left[\begin{array}{cc}
\Lambda(\zeta) & 0 \\
0 & -\Theta(1-\zeta)
\end{array}\right] z^{\prime}(\zeta) \\
& \mathcal{D}(\tilde{A})=\left(z \in H^{1}\left((0,1) ; \mathbb{C}^{d}\right) \mid z(1)=\tilde{K}^{-1} \tilde{Q} z(0)\right\}
\end{aligned}
$$

generates a $C_{0}$-semigroup, but not a $C_{0}$-group. In particular, $Q_{1}:=\tilde{K}^{-1} \tilde{Q}$ is singular.

Since $A$ is a discrete Riesz spectral operator, due to Lemma [5.6, there is a $K>0$ such that in the ball with radius $K|\operatorname{Re} s|^{2}$ around every eigenvalue $s$ of $A$ lie at most $d$ eigenvalues. Thus there exist sequences $\left(t_{n}\right)_{n \in \mathbb{N}} \subset \mathbb{R}$ and $\left(r_{n}\right)_{n \in \mathbb{N}} \subset(0, \infty)$ with $t_{n} \rightarrow-\infty$ and $r_{n} \rightarrow \infty$ such that the ball with center $t_{n}$ and radius $r_{n}$ lie in $\rho(A)$. By Lemma 3.3 we get

$$
\left\|\left(t_{n}-\tilde{A}\right)^{-1}\right\| \rightarrow 0 .
$$

However, for the operator $\tilde{A}$ the Lemma 5.5, is applicable which implies that $\left\|\left(t_{n}-\tilde{A}\right)^{-1}\right\| \rightarrow \infty$. This leads to a contradiction.

\section{$6 \quad$ Examples}

We consider the one-dimensional wave equation with boundary feedback as in XW11, but we allow for spatial dependent mass density and Young's modulus, given by

$$
\begin{aligned}
w_{t t}(\zeta, t) & =\frac{1}{\rho(\zeta)} \frac{\partial}{\partial \zeta}\left(T(\zeta) w_{\zeta}(\zeta, t)\right), \quad \zeta \in[0,1], t \geq 0 \\
w(0, t) & =0 \\
u(t) & =T(1) w_{\zeta}(1, t) \\
y(t) & =w_{t}(1, t), \\
u(t) & =-\kappa y(t), \kappa>0,
\end{aligned}
$$

where $\zeta \in[0,1]$ is the spatial variable, $w(\zeta, t)$ describes the displacement of the point $\zeta$ of the string at time $t, T(\zeta)>0$ is the Young's modulus of the string, $\rho(\zeta)>0$ is the mass density, and $\kappa>0$. We model this system as a portHamiltonian system. Therefore we introduce the state variable $x=\left[\begin{array}{l}x_{1}(\zeta, t) \\ x_{2}(\zeta, t)\end{array}\right]$ with $x_{1}=\rho(\zeta) \frac{\partial w}{\partial t}$ (momentum), $x_{2}=\frac{\partial w}{\partial \zeta}$ (strain) and the state space $X=$ $L^{2}\left((0,1) ; \mathbb{C}^{d}\right)$. Since the meaning of $w(0, t)=0$ is that in the point $\zeta=0$ the string is fixed for all times, we model this boundary condition as $\frac{\partial w}{\partial t} w(0, t)=0$. Then the closed-loop system (27) can equivalently be written as

$$
\begin{aligned}
\frac{\partial}{\partial t}\left[\begin{array}{l}
x_{1}(\zeta, t) \\
x_{2}(\zeta, t)
\end{array}\right] & =P_{1} \frac{\partial}{\partial \zeta}\left(\mathcal{H}(\zeta)\left[\begin{array}{l}
x_{1}(\zeta, t) \\
x_{2}(\zeta, t)
\end{array}\right]\right) \\
0 & =\left[\begin{array}{llll}
0 & 0 & 1 & 0 \\
\kappa & 1 & 0 & 0
\end{array}\right]\left[\begin{array}{l}
\mathcal{H}(1) x(1, t) \\
\mathcal{H}(0) x(0, t)
\end{array}\right]
\end{aligned}
$$


where $P_{1}=\left[\begin{array}{ll}0 & 0 \\ 0 & 1\end{array}\right], \mathcal{H}(\zeta)=\left[\begin{array}{cc}\frac{1}{\rho(\zeta)} & 0 \\ 0 & T(\zeta)\end{array}\right], W_{1}=\left[\begin{array}{ll}0 & 0 \\ \kappa & 1\end{array}\right], W_{0}=\left[\begin{array}{ll}1 & 0 \\ 0 & 0\end{array}\right]$ and $\kappa>0$. We define the corresponding port-Hamiltonian operator $A$

$$
\begin{aligned}
A x & :=P_{1} \frac{\partial}{\partial \zeta}(\mathcal{H} x), \\
\mathcal{D}(A) & =\left\{x \in X \mid \mathcal{H} x \in H^{1}\left((0,1) ; \mathbb{C}^{2}\right) \text { and }\left[W_{1} \quad W_{0}\right]\left[\begin{array}{l}
(\mathcal{H} x)(1) \\
(\mathcal{H} x)(0)
\end{array}\right]=0\right\}
\end{aligned}
$$

and remind that due to JZ12, Theorem 13.2.2] the system has a unique mild solution if $A$ generates a $C_{0}$-semigroup. To show that the port-Hamiltonian operator $A$ is a discrete Riesz spectral operator, it is sufficient due to Theorem 2.8 to prove that $A$ generates a $C_{0}$-group. So we have only to check that $W_{1} \mathcal{H}(1) Z^{+}(1) \oplus W_{0} \mathcal{H}(0) Z^{-}(0)=\mathbb{C}^{d}$ and $W_{1} \mathcal{H}(1) Z^{-}(1) \oplus W_{0} \mathcal{H}(0) Z^{+}(0)=\mathbb{C}^{d}$, where $Z^{+}(\zeta)$ denotes the span of the eigenvectors of $P_{1} \mathcal{H}(\zeta)$ corresponding to the positive eigenvalues of $P_{1} \mathcal{H}(\zeta)$ and $Z^{-}(\zeta)$ is the span of the eigenvectors of $P_{1} \mathcal{H}(\zeta)$ corresponding to the negative eigenvalues of $P_{1} \mathcal{H}(\zeta)$. Defining $\gamma=$ $\sqrt{T(\zeta) / \rho(\zeta)}$, the matrix function $P_{1} \mathcal{H}$ can be factorized as

$$
P_{1} \mathcal{H}=\underbrace{\left[\begin{array}{cc}
\gamma & -\gamma \\
\rho^{-1} & \rho^{-1}
\end{array}\right]}_{S^{-1}} \underbrace{\left[\begin{array}{cc}
\gamma & 0 \\
0 & -\gamma
\end{array}\right]}_{\Delta} \underbrace{\left[\begin{array}{ll}
(2 \gamma)^{-1} & \rho / 2 \\
(2 \gamma)^{-1} & \rho / 2
\end{array}\right]}_{S} .
$$

It is easy to see that $Z^{+}(\zeta)=\operatorname{span}\left[\begin{array}{c}T(\zeta) \\ \gamma(\zeta)\end{array}\right]$ and $Z^{-}(\zeta)=\operatorname{span}\left[\begin{array}{c}-T(\zeta) \\ \gamma(\zeta)\end{array}\right]$. Then it holds for $\kappa \neq-\frac{T(1)}{\gamma(1)}$ and $\kappa \neq \frac{T(1)}{\gamma(1)}$

$$
\begin{aligned}
& W_{1} \mathcal{H}(1) Z^{+}(1) \oplus W_{0} \mathcal{H}(0) Z^{-}(0)=\left[\begin{array}{c}
0 \\
\kappa \gamma(1)+T(1)
\end{array}\right] \oplus\left[\begin{array}{c}
-\gamma(0) \\
0
\end{array}\right]=\mathbb{C}^{2}, \\
& W_{1} \mathcal{H}(1) Z^{-}(1) \oplus W_{0} \mathcal{H}(0) Z^{+}(0)=\left[\begin{array}{c}
0 \\
-\kappa \gamma(1)+T(1)
\end{array}\right] \oplus\left[\begin{array}{c}
\gamma(0) \\
0
\end{array}\right]=\mathbb{C}^{2},
\end{aligned}
$$

Thus, $A$ is a discrete Riesz spectral operator for $\kappa \neq-\frac{T(1)}{\gamma(1)}$ and $\kappa \neq \frac{T(1)}{\gamma(1)}$.

In contrast to XW11 we do not need determine the eigenvalues exactly, which is only possible if $\rho$ and $T$ are constant. For spacial varying coefficients like $\rho(\zeta)=e^{\zeta}$ and $T(\zeta)=\zeta+1$ the problem is not analytically solvable, see [JZ12, Exercise 12.1].

\subsection{Timoshenko-beam}

In JZ12 it is shown that the Timoshenko beam with boundary damping can be formulated as port-Hamiltonian system: It can be written in the form of (12), with $P_{1}=\left[\begin{array}{llll}0 & 1 & 0 & 0 \\ 1 & 0 & 0 & 0 \\ 0 & 0 & 0 & 1 \\ 0 & 0 & 1 & 0\end{array}\right], \mathcal{H}(\zeta)=\left[\begin{array}{cccc}K(\zeta) & 0 & 0 & 0 \\ 0 & \frac{1}{\rho(\zeta)} & 0 & 0 \\ 0 & 0 & E I(\zeta) & 1 \\ 0 & 0 & 0 & \frac{1}{I \rho(\zeta)}\end{array}\right]$ and $P_{0}=\left[\begin{array}{cccc}0 & 0 & 0 & -1 \\ 0 & 0 & 0 & 0 \\ 0 & 0 & 0 & 0 \\ 1 & 0 & 0 & 0\end{array}\right]$, where 
the $K(\zeta)$ denotes the shear modulus, $E I(\zeta)$ is the product of Young's modulus of elasticity and the moment of inertia of a cross section, $\rho(\zeta)$ is the mass per unit length and $I_{\rho}(\zeta)$ denotes the rotary moment of inertia of a cross section. All these physical parameters are positive and continuously differentiable functions of $\zeta$. To model the fact that the beam is clamped in at $\zeta=0$ and controlled at $\zeta=1$ by the force and moment feedback, we add the boundary condition $\left[\begin{array}{l}0 \\ 0 \\ 0 \\ 0\end{array}\right]=\left[\begin{array}{ll}W_{1} W_{0}\end{array}\right]\left[\begin{array}{l}(\mathcal{H}(x)(1, t) \\ (\mathcal{H}(x)(0, t)\end{array}\right]$ with $\left[W_{1} W_{0}\right]=\left[\begin{array}{cccccccc}0 & 0 & 0 & 0 & 0 & 1 & 0 & 0 \\ 0 & 0 & 0 & 0 & 0 & 0 & 0 \\ 1 & \alpha_{1} & 0 & 0 & 0 & 0 & 0 & 0 \\ 0 & 0 & 1 & \alpha_{2} & 0 & 0 & 0 & 0\end{array}\right]$ and $\alpha_{1}, \alpha_{2}$ are given positive gain feedback constants.

$\mathrm{Xu}$ and Feng dedicate the paper XF02 to this example and they proved under the extra assumption that all physical constants are independent of $\zeta$ that the eigenvectors and generalized eigenvectors of the operator form a Riesz basis. This example is also revisited in [Vil07] using another approach. Using our main theorem, we can easy verify that the associated system operator is a discrete Riesz spectral operator.

\section{Closing remarks and open problems}

We have shown that a port-Hamiltonian system of the form (11) is a Riesz spectral operator if and only if it generates a $C_{0}$-group. Many (hyperbolic) systems can be written into this form, with as main exception the Euler-Bernoulli beam equation. Of course the basis property of this equation is well-studied, and many results are known, see e.g. GW19. However, we assert that the main result of this paper does not hold for the Euler-Bernoulli beam equation.

In Theorem 2.8 we have shown that if the port-Hamiltonian systems (1) is a Riesz spectral operator, then the eigenvalues (counted according to the algebraic multiplicity) can be decomposed into finitely many sets each having a uniform gap. If we count the eigenvalues without multiplicity, then [JZ01a, Theorem 2] shows that the eigenvalues can be decomposed into at most $d$ sets each having a uniform gap. We claim that this results holds true if we count the eigenvalues according to the algebraic multiplicity.

One may ask whether the main theorem of this paper (Theorem 2.8) holds if we drop the assumption that $P_{0}$ is skew-symmetric. Our proof uses the fact that every well-posed port-Hamiltonian control system (12) is exactly controllable in finite time and this property is only known in the case that $P_{0}$ is skew-symmetric. Thus, if $P_{0}$ is an arbitrary $d \times d$-matrix, then our proof carries over to this more general case, provided we add the assumption that the corresponding portHamiltonian system is exactly controllable in finite-time. However, we assert that even when $P_{0}$ is not skew-symmetric, the system (12) is exactly controllable in finite time, and thus this extra assumption would not be needed.

B.-Z. Guo and J.-M. Wang GW19] studied the Riesz basis property for a closely related class of systems, that is, hyperbolic systems of the form $\frac{\partial x}{\partial t}=$ $K(\zeta) \frac{\partial x}{\partial \zeta}+C(\zeta) x$ with $K$ and $C$ diagonal. They showed that for their class of systems the state space can be split into two parts, one part generated by a $C_{0^{-}}$ group and the other generated by an operator without spectrum, see GW19, 
Theorem 4.10]. In particular, the spectrum of their operators always lie in a strip parallel to the imaginary axis. This result does not generalize to our system class as the following example shows.

Example 7.1. We consider the port-Hamiltonian system

$$
\begin{aligned}
\frac{\partial}{\partial t}\left[\begin{array}{l}
x_{1}(\zeta, t) \\
x_{2}(\zeta, t)
\end{array}\right] & =\left[\begin{array}{ll}
1 & 0 \\
0 & \frac{1}{2}
\end{array}\right] \frac{\partial}{\partial \zeta}\left[\begin{array}{l}
x_{1}(\zeta, t) \\
x_{2}(\zeta, t)
\end{array}\right]+\left[\begin{array}{cc}
0 & 1 \\
-1 & 0
\end{array}\right]\left[\begin{array}{l}
x_{1}(\zeta, t) \\
x_{2}(\zeta, t)
\end{array}\right] \\
0 & =\left[\begin{array}{llll}
1 & 0 & 0 & 1 \\
0 & 1 & 0 & 0
\end{array}\right]\left[\begin{array}{l}
x(1, t) \\
x(0, t)
\end{array}\right]
\end{aligned}
$$

The system operator generates a $C_{0}$-semigroup, but there exists a sequence of eigenvalues which real parts converge to $-\infty$.

The following example shows that the equivalence 1) $\Leftrightarrow 2$ ) in Theorem 2.8 does not hold for generators $A$ of $C_{0}$-semigroups $(T(t))_{t \geq 0}$ on Hilbert spaces even if we additionally assume that there exists admissible control operator $B \in \mathcal{L}\left(\mathbb{C}^{d}, X_{-1}\right)$ for $(T(t))_{t \geq 0}$ such that the control system $\dot{x}(t)=A x(t)+B u(t)$ is exactly controllable in finite time.

Example 7.2. Let $A: \mathcal{D}(A) \subset \ell^{2} \rightarrow \ell^{2}$ be defined by $(A x)_{n}=\left(s_{n} x_{n}\right)_{n}$, $\left(s_{n}\right)_{n \in \mathbb{N}}=\left(-2^{n}\right)_{n \in \mathbb{N}}$, and $\mathcal{D}(A)=\left\{\left.x \in \ell^{2}(\mathbb{N})\left|\sum_{n \in \mathbb{N}}\left(1+\left|s_{n}\right|^{2}\right)\right| x_{n}\right|^{2}<\infty\right\}$. Clearly, $A$ is a discrete Riesz spectral operator, generates a $C_{0}$-semigroup, but not a $C_{0}$-group. Here $X_{-1}=\ell_{-1}^{2}=\left\{\left(x_{n}\right)_{n \in \mathbb{N}} \mid \sum_{n \in \mathbb{N}} \frac{\left|x_{n}\right|^{2}}{\left(1+\left|s_{n}\right|^{2}\right)}<\infty\right\}$. Hence, we can identify $B \in \mathcal{L}\left(\mathbb{C}, \ell_{-1}^{2}\right)$ with a sequence $\left(b_{n}\right) \in \ell_{-1}^{2}$. Let $\left(b_{n}\right)_{n \in \mathbb{N}}=$ $\left(\sqrt{2^{n}}\right)_{n \in \mathbb{N}}$. Then it holds $\left(b_{n}\right)_{n \in \mathbb{N}} \in \ell_{-1}^{2}$. The Carleson measure criterion of Weiss Wei88 implies the admissibility of $B$. Exact controllability in finite time follows from [JZ01b, Theorem 2].

\section{References}

[AI95] S. A. Avdonin and S. A. Ivanov. Families of Exponentials. Cambridge University Press, Cambridge, 1995.

[AJ14] B. Augner and B. Jacob. Stability and stabilization of infinitedimensional linear port-Hamiltonian systems. Evol. Equ. Control Theory, 3:207-229, 2014.

[Aug16] B. Augner. Stabilisation of Infinite-dimensional Port-Hamiltonian System. PhD thesis, University of Wuppertal, 2016.

[Cur84] R. F. Curtain. Spectral systems. Internat. J. Control, 39(4):657666, 1984.

[CZ95] R. F. Curtain and H. Zwart. An Introduction to InfiniteDimensional Linear Systems Theory, volume 21 of Texts in Applied Mathematics. Springer-Verlag, New York, 1995. 
[CZ20] R. F. Curtain and H. Zwart. Introduction to Infinite-Dimensional Systems Theory, volume 71 of Texts in Applied Mathematics. Springer-Verlag, New York, 2020.

[DMSB09] V. Duindam, A. Macchelli, S. Stramigioli, and H. Bruyninckx, editors. Modeling and Control of Complex Physical Systems. SpringerVerlag, Berlin, 2009.

[DS71] N. Dunford and J. T. Schwartz. Linear Operators. Part III: Spectral Operators. Interscience Publishers [John Wiley \& Sons, Inc.], New York, 1971.

[EMvdS07] D. Eberard, B. M. Maschke, and A. J. van der Schaft. An extension of Hamiltonian systems to the thermodynamic phase space: towards a geometry of nonreversible processes. Rep. Math. Phys., 60(2):175-198, 2007.

[EN00] K.-J. Engel and R. Nagel. One-Parameter Semigroups for Linear Evolution Equations, volume 194 of Graduate Texts in Mathematics. Springer-Verlag, New York, 2000.

[GGK90] I. Gohberg, S. Goldberg, and M. A. Kaashoek. Classes of Linear Operators. Vol. I, volume 49 of Operator Theory: Advances and Applications. Birkhäuser Verlag, Basel, 1990.

[GW19] B.-Z. Guo and J.-M. Wang. Control of Wave and Beam PDEs. Communications and Control Engineering Series. Springer, Cham, 2019. The Riesz Basis Approach.

[GX04] B.-Z. Guo and G.-Q. Xu. Riesz bases and exact controllability of $C_{0}$-groups with one-dimensional input operators. Systems Control Lett., 52(3-4):221-232, 2004.

[GZ01] B. Guo and H. Zwart. Riesz spectral systems. MEMORANDUM, No. 1594, 2001.

[HP18] J.-P. Humaloja and L. Paunonen. Robust regulation of infinitedimensional port-Hamiltonian systems. IEEE Trans. Automat. Control, 63(5):1480-1486, 2018.

[JK19a] B. Jacob and J. T. Kaiser. On exact controllability of infinitedimensional linear port-Hamiltonian systems. IEEE Control Systems Letters, 3(3):661-666, 2019.

[JK19b] B. Jacob and J. T. Kaiser. Well-posedness of systems of 1-D hyperbolic partial differential equations. J. Evol. Equ., 19(1):91-109, 2019.

[JMZ15] B. Jacob, K. Morris, and H. Zwart. $C_{0}$-semigroups for hyperbolic partial differential equations on a one-dimensional spatial domain. J. Evol. Equ., 15(2):493-502, 2015. 
[JZ99] B. Jacob and H. Zwart. Equivalent conditions for stabilizability of infinite-dimensional systems with admissible control operators. SIAM J. Control Optim., 37(5):1419-1455, 1999.

[JZ01a] B. Jacob and H. Zwart. Exact observability of diagonal systems with a finite-dimensional output operator. Systems Control Lett., 43(2):101-109, 2001.

[JZ01b] B. Jacob and H. Zwart. Exact observability of diagonal systems with a one-dimensional output operator. Int. J. Appl. Math. Comput. Sci., 11(6):1277-1283, 2001.

[JZ12] B. Jacob and H. J. Zwart. Linear port-Hamiltonian Systems on Infinite-Dimensional Spaces, volume 223 of Operator Theory: Advances and Applications. Birkhäuser/Springer Basel AG, Basel, 2012. Linear Operators and Linear Systems.

[JZ18] B. Jacob and H. Zwart. An operator theoretic approach to infinitedimensional control systems. GAMM-Mitt., 41(4):e201800010, 14, 2018 .

[LGZM05] Y. Le Gorrec, H. Zwart, and B. Maschke. Dirac structures and boundary control systems associated with skew-symmetric differential operators. SIAM J. Control Optim., 44:1864-1892, 2005.

[LW83] J.-C. Louis and D. Wexler. On exact controllability in Hilbert spaces. J. Differential Equations, 49(2):258-269, 1983.

[RLGMZ14] H. Ramírez, Y. Le Gorrec, A. Macchelli, and H. Zwart. Exponential stabilization of boundary controlled port-Hamiltonian systems with dynamic feedback. IEEE Trans. Automat. Control, 59(10):2849-2855, 2014.

[RW94] D. L. Russell and G. Weiss. A general necessary condition for exact observability. SIAM J. Control Optim., 32(1):1-23, 1994.

[RZLG17] H. Ramírez, H. Zwart, and Y. Le Gorrec. Stabilization of infinite dimensional port-Hamiltonian systems by nonlinear dynamic boundary control. Automatica J. IFAC, 85:61-69, 2017.

[Sta05] O. Staffans. Well-Posed Linear Systems, volume 103 of Encyclopedia of Mathematics and its Applications. Cambridge University Press, Cambridge, 2005.

[SZ18] J. Schmid and H. Zwart. Stabilization of port-Hamiltonian systems by nonlinear boundary control in the presence of disturbances. https://arxiv.org/abs/1804.10598v2, 2018.

[Tre00a] C. Tretter. Spectral problems for systems of differential equations $y^{\prime}+A_{0} y=\lambda A_{1} y$ with $\lambda$-polynomial boundary conditions. Math. Nachr., 214:129-172, 2000. 
[Tre00b] C. Tretter. Linear operator pencils $A-\lambda B$ with discrete spectrum. Integral Equations Operator Theory, 37(3):357-373, 2000.

[TW09] M. Tucsnak and G. Weiss. Observation and Control for Operator Semigroups. Birkhäuser Advanced Texts: Basler Lehrbücher. Birkhäuser Verlag, Basel, 2009.

[TW14] M. Tucsnak and G. Weiss. Well-posed systems - the LTI case and beyond. Automatica J. IFAC, 50(7):1757-1779, 2014.

[vdS06] A. van der Schaft. Port-Hamiltonian systems: an introductory survey. In International Congress of Mathematicians. Vol. III, pages 1339-1365. Eur. Math. Soc., Zürich, 2006.

[Vil07] J. Villegas. A Port-Hamiltonian Approach to Distributed Parameter Systems. PhD thesis, University of Twente, Netherlands, 2007.

[Was87] W. Wasow. Asymptotic Expansions for Ordinary Differential Equations. Dover Publications, Inc., New York, 1987. Reprint of the 1976 edition.

[Wei88] G. Weiss. Admissibility of input elements for diagonal semigroups on $l^{2}$. Systems Control Lett., 10(1):79-82, 1988.

[XF02] G.-Q. Xu and D.-X. Feng. The Riesz basis property of a Timoshenko beam with boundary feedback and application. IMA J. Appl. Math., 67(4):357-370, 2002.

[XG03] G.-Q. Xu and B.-Z. Guo. Riesz basis property of evolution equations in Hilbert spaces and application to a coupled string equation. SIAM J. Control Optim., 42(3):966-984, 2003.

[XW11] C.-Z. Xu and G. Weiss. Eigenvalues and eigenvectors of semigroup generators obtained from diagonal generators by feedback. Commun. Inf. Syst., 11(1):71-104, 2011.

[ZLMV10] H. Zwart, Y. Le Gorrec, B. Maschke, and J. Villegas. Wellposedness and regularity of hyperbolic boundary control systems on a one-dimensional spatial domain. ESAIM Control Optim. Calc. Var., 16(4):1077-1093, 2010.

[Zwa10] H. Zwart. Riesz basis for strongly continuous groups. J. Differential Equations, 249(10):2397-2408, 2010. 